This item was submitted to Loughborough's Research Repository by the author.

Items in Figshare are protected by copyright, with all rights reserved, unless otherwise indicated.

\title{
Jordan-Kronecker invariants of finite-dimensional Lie algebras
}

PLEASE CITE THE PUBLISHED VERSION

VERSION

AM (Accepted Manuscript)

\section{PUBLISHER STATEMENT}

This work is made available according to the conditions of the Creative Commons Attribution-NonCommercialNoDerivatives 4.0 International (CC BY-NC-ND 4.0) licence. Full details of this licence are available at: https://creativecommons.org/licenses/by-nc-nd/4.0/

\section{LICENCE}

CC BY-NC-ND 4.0

\section{REPOSITORY RECORD}

Bolsinov, Alexey V., A. Vorontsov, Pumei Zhang, D. Dowell, I. Kozlov, and Anton Izosimov. 2019. “Jordankronecker Invariants of Finite-dimensional Lie Algebras”. figshare. https://hdl.handle.net/2134/23041. 


\title{
Jordan-Kronecker invariants of finite-dimensional Lie algebras
}

\author{
Alexey Bolsinov \\ Loughborough University \\ (joint with A. Vorontsov, P. Zhang, D. Dowell, I. Kozlov and \\ A. Izosimov) \\ Integrability, Recursion, Geometry And Mechanics \\ Celebrating Franco Magri's 70th birthday \\ September 5-9, 2016 at RISM - Villa Toeplitz - Varese
}




\section{Outline}

- Motivation and Generalised Argument Shift Conjecture

- Jordan-Kronecker decomposition theorem

- Jordan-Kronecker invariants of a finite-dimensional Lie algebra

- General facts about JK invariants

- Polynomiality of the algebra of $\mathrm{Ad}^{*}$-invariants

- Examples 


\section{Motivation}

- It is a very natural idea.

A Lie algebra is defined by its structure tensor $c_{i j}^{k}$. Too complicated!

Classical approach: Take ad $\xi=\sum_{i} c_{i j}^{k} \xi^{i}$ for generic $\xi \in \mathfrak{g}$ and then study the properties of this operator.

Another option: $\quad$ Take a form $\mathcal{A}_{x}=\sum_{k} c_{i j}^{k} x_{k}$ for generic $x \in \mathfrak{g}^{*}$.

Unfortunately, the only invariant is the rank of rank $\mathcal{A}_{x}$. But... non-trivial invariants appear if we consider a pair of forms $\mathcal{A}_{x}$ and $\mathcal{A}_{a}, x, a \in \mathfrak{g}^{*}$. Can we get anything interesting?

- Many classical facts become more transparent and some new results can be derived.

- Arbitrary Lie algebras, not necessarily semisimple.

- Generalised argument shift conjecture 


\section{Historical remarks}

1. The first and main ingredient is the argument shift method suggested by A. Mischenko and A. Fomenko in 1976 as a generalisation of S. Manakov construction.

2. In 1988, I. Gelfand and I. Zakharevich observed a very important relationship between compatible Poisson brackets and Jordan-Kronecker decomposition theorem and used it to study the so-called micro-Kronecker pencils and their applications.

3. Jordan-Kronecker decomposition for a pencil of skew-symmetric forms (C. Jordan, K. Weierstrass, L. Kronecker, F. Gantmacher, G. Gurevich, R. Thompson, ... ).

4. Transition from algebraic canonical forms of pencils in linear algebra to normal forms of compatible Poisson brackets was done by F.-J. Turiel (series of papers since 1989).

5. The idea of JK invariants was conceptualised in the framework of an informal Moscow-Loughborough research seminar for PhD students (A. Izosimov, P. Zhang, A. Konjaev, A. Vorontsov, I. Kozlov, D. Dowell).

6. This talk is based on our joint paper with Pumei Zhang. 


\section{Conjecture}

In the two forms $\mathcal{A}_{x} \mathcal{A}_{a}$, one can easily recognise two famous compatible Poisson structures on the dual space $\mathfrak{g}^{*}$ of a Lie algebra $\mathfrak{g}$.

The first is the standard Lie-Poisson bracket:

$$
\{f, g\}(x)=\mathcal{A}_{x}(d f(x), d g(x))=\sum\left(c_{i j}^{k} x_{k}\right) \frac{\partial f}{\partial x_{i}} \frac{\partial g}{\partial x_{j}} .
$$

The second is:

$$
\{f, g\}_{a}(x)=\mathcal{A}_{a}(d f(x), d g(x))=\sum\left(c_{i j}^{k} a_{k}\right) \frac{\partial f}{\partial x_{i}} \frac{\partial g}{\partial x_{j}}, \quad a \in \mathfrak{g}^{*} \text { (fixed). }
$$

Consider the following family of functions on $\mathfrak{g}^{*}$ (algebra of shifts):

$$
\mathcal{F}_{a}=\{f(x+\lambda a) \mid f \in \mathrm{I}(\mathfrak{g}), \lambda \in \mathbb{R}\} .
$$

Theorem (Mischenko, Fomenko)

1) $\mathcal{F}_{a}$ is commutative w.r.t. both $\{$,$\} and \{,\}_{a}$.

2) If $\mathfrak{g}$ is semisimple, then $\mathcal{F}_{a}$ is complete.

Mischenko-Fomenko conjecture.

For any $\mathfrak{g}$, there is a complete commutative family $\mathcal{G} \subset P(\mathfrak{g})$ of polynomials in involution. 


\section{Conjecture}

In the two forms $\mathcal{A}_{x}$ and $\mathcal{A}_{a}$, one can easily recognise two famous compatible Poisson structures on the dual space $\mathfrak{g}^{*}$ of a Lie algebra $\mathfrak{g}$. The first is the standard Lie-Poisson bracket:

$$
\{f, g\}(x)=\mathcal{A}_{x}(d f(x), d g(x))=\sum\left(c_{i j}^{k} x_{k}\right) \frac{\partial f}{\partial x_{i}} \frac{\partial g}{\partial x_{j}} .
$$

The second is:

$$
\{f, g\}_{a}(x)=\mathcal{A}_{x}(d f(x), d g(x))=\sum\left(c_{i j}^{k} a_{k}\right) \frac{\partial f}{\partial x_{i}} \frac{\partial g}{\partial x_{j}}, \quad a \in \mathfrak{g}^{*} \text { (fixed). }
$$

Consider the following family of functions on $\mathfrak{g}^{*}$ (algebra of shifts):

$$
\mathcal{F}_{a}=\{f(x+\lambda a) \mid f \in \mathrm{I}(\mathfrak{g}), \lambda \in \mathbb{R}\} .
$$

Theorem (Mischenko, Fomenko)

1) $\mathcal{F}_{a}$ is commutative w.r.t. both $\{$,$\} and \{,\}_{a}$.

2) If $\mathfrak{g}$ is semisimple, then $\mathcal{F}_{a}$ is complete.

\section{Generalised Argument Shift Conjecture.}

For any $\mathfrak{g}$, there is a complete commutative family $\mathcal{G}_{a} \subset P(\mathfrak{g})$ of polynomials in bi-involution. 


\section{Jordan-Kronecker decomposition}

This theorem gives a classification of skew-symmetric bilinear forms $\mathcal{A}, \mathcal{B}$ on a finite-dimensional vector space by reducing them simultaneously to an elegant block-diagonal form.

\section{Theorem}

Let $\mathcal{A}$ and $\mathcal{B}$ be two skew-symmetric bilinear forms on a complex vector space $V$. Then by an appropriate choice of a basis, their matrices can be simultaneously reduced to the following canonical block-diagonal form:

$$
\mathcal{A} \mapsto\left(\begin{array}{cccc}
\mathcal{A}_{1} & & & \\
& \mathcal{A}_{2} & & \\
& & \ddots & \\
& & & \mathcal{A}_{k}
\end{array}\right), \quad \mathcal{B} \mapsto\left(\begin{array}{cccc}
\mathcal{B}_{1} & & & \\
& \mathcal{B}_{2} & & \\
& & \ddots & \\
& & & \mathcal{B}_{k}
\end{array}\right)
$$

where the pairs of the corresponding blocks $\mathcal{A}_{i}$ and $\mathcal{B}_{i}$ can be of the following three types: 


\section{Jordan-Kronecker decomposition}

$\mathcal{A}_{i}$

Jordan block

$\left(\lambda_{i} \in \mathbb{C}\right)$

Jordan block

$\left(\lambda_{i}=\infty\right)$

Kronecker block
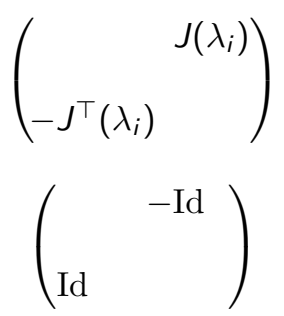

$\mathcal{B}_{i}$

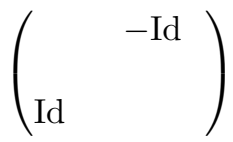

$\left(\begin{array}{rr} & J(0) \\ -J^{\top}(0) & \end{array}\right)$
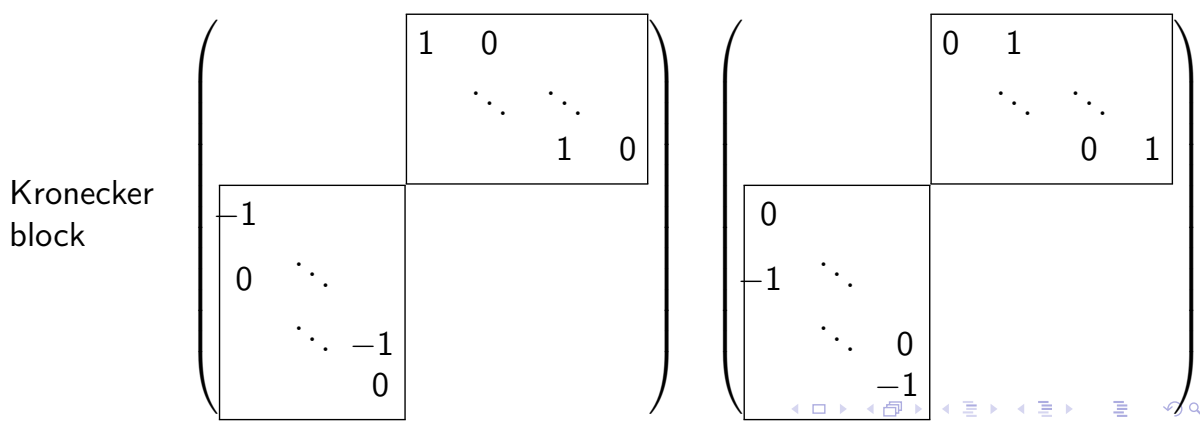


\section{Some comments}

- Characteristic numbers $\lambda_{i}$ play the same role as "eigenvalues" in the case of linear operators (recursion operators). More precisely, $\lambda_{i}$ are those numbers for which the rank of $\mathcal{A}_{\lambda}=\mathcal{A}+\lambda \mathcal{B}$ for $\lambda=\lambda_{i}$ is not maximal.

- If $\mu \neq \lambda_{i}$, then $\mathcal{A}_{\mu}=\mathcal{A}+\mu \mathcal{B}$ is called regular (in the pencil $\left.\mathcal{P}=\left\{\mathcal{A}_{\lambda}\right\}\right)$.

- Characteristic numbers are the roots of the characteristic polynomial $\mathrm{f}_{\mathcal{P}}(\lambda)=\operatorname{gcd}\left\{\operatorname{Pf}_{i_{1} \ldots i_{2 k}}(\mathcal{A}+\lambda \mathcal{B})\right\}, 2 k=\operatorname{rank} \mathcal{P}$.

- The sizes of Kronecker blocks are odd $2 k_{i}-1$, the sizes of Jordan blocks are even $2 j_{m}$. The numbers $k_{i}$ and $j_{m}$ are called Kronecker and Jordan indices of the pencil.

- The Jordan-Kronecker decomposition theorem implies the existence of a large subspace which is isotropic simultaneously for all forms from the pencil $\mathcal{P}$ (bi-Lagrangian subspace). 


\section{Some more comments}

\section{Theorem}

For every pencil $\mathcal{P}=\left\{\mathcal{A}_{\lambda}\right\}$, there is a bi-Lagrangian subspace $L \subset V$. This means that $L$ is isotropic with respect to all $\mathcal{A}_{\mu} \in \mathcal{P}$ and is maximal isotropic for all regular forms $\mathcal{A}_{\lambda} \in \mathcal{P}$. In particular, $\operatorname{dim} L=\frac{1}{2}(\operatorname{dim} V+\operatorname{corank} \mathcal{P})$.

There is one very special bi-isotropic subspace of $V$ :

$$
L_{\text {can }}=\sum_{\lambda} \operatorname{Ker} \mathcal{A}_{\lambda},
$$

\section{Proposition}

- $L_{\text {can }}$ is contained in any bi-Lagrangian subspace $L$ and is the intersection of all bi-Lagrangian subspaces.

- $\operatorname{dim} L_{\text {can }}=\sum_{i=1}^{s} k_{i}=\frac{1}{2}(\operatorname{dim} V+\operatorname{corank} \mathcal{P})-\operatorname{deg} f_{\mathcal{P}}$

- $\operatorname{dim} L_{\text {can }}$ is bi-Lagrangian if and only if $\mathcal{P}$ is of pure Kronecker type. 


\section{Definition of Jordan-Kronecker invariants}

Let $\mathfrak{g}$ be a Lie algebra and $\mathfrak{g}^{*}$ its dual space. We say that $(x, a) \in \mathfrak{g}^{*} \times \mathfrak{g}^{*}$ is a generic pair if the type of the Jordan-Kronecker decomposition of $\mathcal{A}_{x}=\sum_{k} c_{i j}^{k} x_{k}$ and $\mathcal{A}_{a}=\sum_{k} c_{i j}^{k} a_{k}$ is the same for all points in the neighborhoods of $(x, a)$.

Definition

The type of the Jordan-Kronecker canonical form for the pencil $\mathcal{A}_{x}+\lambda \mathcal{A}_{a}$ for a generic pair $(x, a) \in \mathfrak{g}^{*}$, is called the Jordan-Kronecker invariant of $\mathfrak{g}$. In particular, a Lie algebra $\mathfrak{g}$ is

- of Kronecker type,

- of Jordan (symplectic) type,

- of mixed type,

if the Jordan-Kronecker decomposition of a (generic) pencil $\mathcal{A}_{x}+\lambda \mathcal{A}_{a}$ consists of

- only Kronecker blocks,

- only Jordan blocks

- both Jordan and Kronecker blocks.

The Kronecker and Jordan indices of a generic pencil $\left\{\mathcal{A}_{x}+\lambda \mathcal{A}_{a}\right\}$ are said to be the Kronecker and Jordan indices of $\mathfrak{g}$. 


\section{Prerequisites: basic notions}

- Finite-dimensional Lie algebra $\mathfrak{g}$ and its dual space $\mathfrak{g}^{*}$

- Lie-Poisson bracket on $\mathfrak{g}^{*}$ :

$$
\{f, g\}(x)=\langle x,[d f(x), d g(x)]\rangle
$$

- Annihilator of $a \in \mathfrak{g}^{*}$ :

$$
\text { Ann } a=\left\{\xi \in \mathfrak{g} \mid \operatorname{ad}_{\xi}^{*} a=0\right\}
$$

- $\mathrm{f}_{\mathfrak{g}}(x)=\operatorname{gcd}\left\{\operatorname{Pf}_{i_{1} \ldots i_{2 k}}\left(\mathcal{A}_{x}\right)\right\}$, the fundamental semi-invariant of $\mathfrak{g}$.

- Index of $\mathfrak{g}$ is the codimension of generic $\mathrm{Ad}^{*}$-orbits:

$$
\text { ind } \mathfrak{g}=\min _{x \in \mathfrak{g}^{*}} \operatorname{dim} \operatorname{Ann} x
$$

If ind $\mathfrak{g}=0$, then the Lie algebra $\mathfrak{g}$ is called Frobenius.

- Singular set

$$
\text { Sing }=\left\{y \in \mathfrak{g}^{*} \mid \operatorname{dim} \operatorname{Ann}(x)>\text { ind } \mathfrak{g}\right\}
$$




\section{Kronecker case}

\section{Theorem}

The following properties of a Lie algebra $\mathfrak{g}$ are equivalent

1. $\mathfrak{g}$ is of Kronecker type, i. e. the Jordan-Kronecker decomposition of the (generic) pencil $\mathcal{A}_{x}+\lambda \mathcal{A}_{a}$ consists of Kronecker blocks only,

2. codim Sing $\geq 2$, where

$$
\text { Sing }=\left\{y \in \mathfrak{g}^{*} \mid \operatorname{dim} \text { Ann } y>\text { ind } \mathfrak{g}\right\} \subset \mathfrak{g}^{*}
$$

is the singular subset of $\mathfrak{g}^{*}$,

3. the algebra of shifts $\mathcal{F}_{a}$ is complete.

\section{Example}

Let $\mathfrak{g}$ be semisimple. Then $\mathfrak{g}$ is of Kronecker type and the Kronecker indices $k_{1}, \ldots, k_{s}, s=$ ind $\mathfrak{g}$, are exactly the degrees of the basic Casimirs $f_{1}, \ldots, f_{s}$ (invariants of the adjoint representation).

This property holds for many other classes of Lie algebras, e. g., $e(n)=s o(n)+\mathbb{R}^{n}$. 


\section{Jordan case}

Theorem

The following properties of a Lie algebra $\mathfrak{g}$ are equivalent

1. $\mathfrak{g}$ is of Jordan type, i.e. the Jordan-Kronecker decomposition of the generic pencil $\mathcal{A}_{x}+\lambda \mathcal{A}_{a}$ consists of Jordan blocks only,

2. a generic form $\mathcal{A}_{x}$ is non-degenerate, i.e., ind $\mathfrak{g}=0$ and $\mathfrak{g}$ is a Frobenius Lie algebra,

3. $\mathcal{F}_{a}$ is trivial, i.e., $\mathcal{F}_{a}=\mathbb{C}$. 


\section{General case}

\section{Proposition}

1) The number of Kronecker blocks in the JK decomposition is equal to the index of $\mathfrak{g}$.

2) The number of trivial Kronecker blocks is greater or equal to the dimension of the center of $\mathfrak{g}$.

3) The number of independent functions in the family of shifts $\mathcal{F}_{a}$ is equal to $\sum k_{i}$ where $k_{i}$ are the Kronecker indices of $\mathfrak{g}$ or, equivalently,

$$
\operatorname{tr} \cdot \operatorname{deg} . \mathcal{F}_{a}=\frac{1}{2}(\operatorname{dim} \mathfrak{g}+\text { ind } \mathfrak{g})-\operatorname{deg} \mathrm{p}_{\mathfrak{g}} \text {. }
$$

Theorem (A. Vorontsov, 2011)

Let $f_{1}(x), f_{2}(x), \ldots, f_{s}(x) \in P(\mathfrak{g})$ be algebraically independent polynomial $\mathrm{Ad}^{*}$-invariants of $\mathfrak{g}, s=$ ind $\mathfrak{g}$, and $m_{1} \leq m_{2} \leq \cdots \leq m_{s}$ be their degrees, $m_{i}=\operatorname{deg} f_{i}$. Then

$$
m_{i} \geq k_{i}
$$

where $k_{1} \leq k_{2} \leq \cdots \leq k_{s}$ are Kronecker indices of the Lie algebra $\mathfrak{g}$. In the semisimple case (but not only!): $m_{i}=k_{i}$. 


\section{Polynomiality of the algebra $\mathrm{I}(\mathfrak{g})$ of $\mathrm{Ad}^{*}$-invariants}

Let $f_{1}, \ldots, f_{s}, s=$ ind $\mathfrak{g}$, be algebraically independent $\mathrm{Ad}^{*}$-invariant polynomials. Then

$$
\sum_{i=1}^{s} \operatorname{deg} f_{i} \geq \sum_{i=1}^{s} k_{i}=\frac{1}{2}(\operatorname{dim} \mathfrak{g}+\operatorname{ind} \mathfrak{g})-\operatorname{deg} \mathfrak{f}_{\mathfrak{g}} .
$$

For many classes of Lie algebras, this estimate becomes an equality (known as a sum rule) which (surprisingly) implies that the differentials $d f_{1}, \ldots, d f_{s}$ are linearly independent at $x \in \mathfrak{g}^{*}$ if and only if $x \notin \operatorname{Sing}_{1}$.

\section{Theorem}

Let $k_{1} \leq \cdots \leq k_{s}$ be the Kronecker indices of $\mathfrak{g}$ and $f_{1}, \ldots, f_{s} \in \mathrm{I}(\mathfrak{g})$ be algebraically independent $\mathrm{Ad}^{*}$-invariant polynomials with $\operatorname{deg} f_{1} \leq \operatorname{deg} f_{2} \leq \cdots \leq \operatorname{deg} f_{s}, s=$ ind $\mathfrak{g}$. Assume that $\mathfrak{g}$ is unimodular and $\mathrm{f}_{\mathfrak{g}} \in \mathrm{I}(\mathfrak{g})$. Then the following conditions are equivalent:

1. $k_{i}=\operatorname{deg} f_{i}, i=1 \ldots, s$;

2. $\sum_{i=1}^{s} \operatorname{deg} f_{i}=\frac{1}{2}(\operatorname{dim} \mathfrak{g}+\operatorname{ind} \mathfrak{g})-\operatorname{deg} f_{\mathfrak{g}}$;

3. $\mathrm{I}(\mathfrak{g})$ is polynomial on $f_{1}, \ldots, f_{s}$. 


\section{Characteristic numbers and singular set}

- The characteristic numbers $\lambda_{i}$ of the Lie algebra $\mathfrak{g}^{*}$ are, by definition, the characteristic numbers for a generic pencil $\mathcal{A}_{x}+\lambda \mathcal{A}_{a}$, $(x, a) \in \mathfrak{g}^{*} \times \mathfrak{g}^{*}$.

- The characteristic numbers $\lambda_{i}=\lambda_{i}(x, a)$ are defined by the following algebraic condition $x+\lambda_{i} a \in$ Sing.

- The characteristic numbers exist if and only if codim Sing $=1$.

- The codimension one component of the singular set Sing is defined by one polynomial equation $f_{\mathfrak{g}}(x)=0$, where $\mathrm{f}_{\mathfrak{g}}(x)$ is the fundamental invariant of $\mathfrak{g}$. The rest of Sing will be denoted by Sing $_{1}$.

- The characteristic numbers of $\mathfrak{g}$ are the roots of $f_{\mathfrak{g}}(x+\lambda a)=0$.

- The characteristic numbers are in bi-involution. 


\section{Frobenius Lie algebras}

Let $\mathfrak{g}$ be Frobenius, i.e., ind $\mathfrak{g}=0$. Then Sing is defined by one polynomial, namely, $\mathrm{f}_{\mathfrak{g}}(x)=\operatorname{Pf}\left(\mathcal{A}_{x}\right)=\sqrt{\operatorname{det}\left(c_{i j}^{k} x_{k}\right)}$. The degree of this polynomial is $\frac{1}{2} \operatorname{dim} \mathfrak{g}$.

\section{Theorem}

Let $\mathfrak{g}$ be a Frobenius Lie algebra, and the (geometric) degree of Sing $\subset \mathfrak{g}^{*}$ be equal to $k=\frac{1}{2} \operatorname{dim} \mathfrak{g}$.

Then a generic pencil $\mathcal{A}_{x}+\lambda \mathcal{A}_{a}$ is diagonalisable (i.e. has no Jordan blocks of size greater than $2 \times 2$ ), all characteristic numbers are distinct, and the coefficients of the "characteristic polynomial" $\mathrm{p}(\lambda)=\operatorname{Pf} \mathcal{A}_{x+\lambda a}$ form a complete family of polynomials in bi-involution. 


\section{Mixed case}

Consider the polynomial $f_{\mathfrak{g}}(x)$, substitute $x+\lambda a$ and consider it as a polynomial in $\lambda$ :

$$
f(\lambda)=f_{\mathfrak{g}}(x+\lambda a)=g_{0}(x)+\lambda g_{1}(x)+\lambda^{2} g_{2}(x)+\cdots+\lambda^{m} g_{m}(x) .
$$

The homogeneous polynomials $g_{0}(x), \ldots, g_{m}(x)$ are obviously the symmetric polynomials of characteristic numbers. So they are in bi-involution and, moreover, they are in bi-involution with the algebra of shifts $\mathcal{F}_{a}$.

Combining the collection of $g_{k}$ 's with the algebra of shifts $\mathcal{F}_{a}$, we obtain an extended algebra of functions in bi-involution $\mathcal{G}_{a}$.

Question. Is $\mathcal{G}_{a}$ complete?

Theorem (A.Isosimov)

The algebra $\mathcal{G}_{a}$ is complete if and only if, the annihilator of a generic singular element is isomorphic to $\mathfrak{a}_{2} \oplus$ centre, where $\mathfrak{a}_{2}$ is the two-dimensional non-Abelian Lie algebra. 


\section{Elashvili conjecture}

Let Ann $a=\left\{\xi \in \mathfrak{g} \mid \operatorname{ad}_{\xi}^{*} a=0\right\}$ be the stationary subalgebra of $a \in \mathfrak{g}^{*}$ with respect to the coadjoint representation. The following estimate is well-known:

$$
\text { ind Ann } a \geq \text { ind } \mathfrak{g}
$$

Elashvili conjecture: if $\mathfrak{g}$ is semisimple, then ind Ann $a=$ ind $\mathfrak{g}$ for all $a \in \mathfrak{g}=\mathfrak{g}^{*}$.

Interpretation in terms of Jordan-Kronecker decomposition:

Proposition

Let $a \in \mathfrak{g}^{*}$ be fixed and $x \in \mathfrak{g}^{*}$ is generic in the sense that the type of the Jordan-Kronecker decomposition of $\mathcal{A}_{x}$ and $\mathcal{A}_{a}$ does not change in a certain neighborhood of $x$. Then

$$
\text { ind Ann } a=\text { ind } \mathfrak{g}
$$

if and only if the Jordan-Kronecker decomposition does not contain any non-trivial Jordan blocks, i.e., the Jordan part is diagonalisable. Otherwise, i.e. if there are non-trivial Jordan blocks, we have strong inequality:

$$
\text { ind Ann } a>\text { ind } \mathfrak{g}
$$




\section{Examples}

- Semisimple Lie algebras:

Pure Kronecker case, the Kronecker indices $k_{i}$ coincide with the degrees of $m_{i}$ of basis Casimirs:

$$
k_{i}=m_{i} .
$$

For classical series these numbers $m_{i}$ are:

- $A_{n}: 2,3,4, \ldots, n+1$;

- $B_{n}: \quad 2,4,6, \ldots, 2 n$;

- $C_{n}: 2,4,6, \ldots, 2 n$;

- $D_{n}: \quad 2,4,6, \ldots, 2 n-2$ and $n$.

- Some semidirect sums.

- $e(n)=s o(n)+\mathbb{R}^{n}:$

JK invariants are the same as those for so $(n+1)$.

- $\mathfrak{g}=s l(n)+\mathbb{R}^{n}:$

Pure Kronecker type with one single Kronecker block, i.e., $k_{1}=\frac{1}{2}(\operatorname{dim} \mathfrak{g}+1)$.

- $\mathfrak{k}+{ }_{\rho} V$ with $\mathfrak{k}$ simple and $\rho$ irreducible:

Pure Kronecker type

(F. Knop, P. Littelmann, B. Priwitzer, AB)

- Lie algebras of low dimension $\leq 5$. The complete list of JK invariants is obtained by $\mathrm{P}$. Zhang. 


\section{Examples}

- Affine Lie algebra afff $(n)=g l(n)+\mathbb{R}^{n}$. The Pfaffian $\operatorname{Pf}\left(\mathcal{A}_{x}\right)=\sqrt{\operatorname{det}\left(c_{i j}^{k} x_{k}\right)}$ is irreducible and we can apply one of above theorems. The pencil $\mathcal{A}_{x}+\lambda \mathcal{A}_{a}$ is of Jordan type, diagonalisable, with $\frac{1}{2} \operatorname{dim} g$ distinct characteristic number. In other words, the Jordan indices of $\mathfrak{g}$ are

$$
\underbrace{1,1, \ldots, 1}_{k}, \quad k=\frac{1}{2}\left(n^{2}+n\right)=\frac{1}{2} \operatorname{dim} \mathfrak{a f f}(n) .
$$

- Another interesting example $\mathfrak{g}=g l(n)+\mathbb{R}^{n^{2}}$, where $\mathbb{R}^{n^{2}}$ is realised as the space of $n \times n$-matrices, and the action of $g l(n)$ on it is left multiplication. The matrix realisation of $\mathfrak{g}$ is: $\left(\begin{array}{ll}A & C \\ 0 & 0\end{array}\right)$.

This Lie algebra is Frobenius, the singular set is defined by $\operatorname{det} C=0$. Hence, we have $n$ distinct characteristic numbers, the multiplicity of each of them is $2 n$, and the Jordan indices are

$$
\underbrace{1,1, \ldots, 1}_{n-2}, 2
$$




\section{Triangular Lie algebra}

Let $t_{n}$ be the Lie algebra of upper triangular $n \times n$ matrices.

The description of Jordan-Kronecker invariants for $\mathfrak{t}_{n}$ easily follows from the results by $A$.Arkhangelskii.

If $n$ is even, then $t_{n}$ is of mixed type. The coadjoint invariants are rational functions $f_{k}=\frac{P_{k}}{Q_{k}}, k=1, \ldots, \frac{n}{2}$ with $\operatorname{deg} P_{k}=k$ and $\operatorname{deg} Q_{k}=k-1$. The Kronecker indices are exactly $\operatorname{deg} P_{k}+\operatorname{deg} Q_{k}$, namely

$$
1,3,5, \ldots, n-1 \text {. }
$$

The singular set Sing $\subset \mathfrak{t}_{n}^{*}$ is defined by an irreducible polynomial $f$ of degree $\frac{n}{2}$. Therefore, $\mathfrak{t}_{n}$ possesses $\frac{n}{2}$ distinct characteristic numbers, each of multiplicity one. In particular, the Jordan part of a generic pencil $\mathcal{A}_{x+\lambda a}$ is diagonalisable and Jordan indices are $1, \ldots, 1$ ( $\frac{n}{2}$ times).

If $n$ is odd, then $t_{n}$ is of Kronecker type and the Kronecker indices are $1,3,5, \ldots, n$.

Important: For all these Lie algebras, the Generalised Argument Shift Conjecture holds. 
Many thanks

for your attention 